\title{
Atributos de hortaliças sob a ótica de consumidores: estudo de caso do pimentão no Distrito Federal
}

\author{
Silvia S Onoyama ${ }^{1}$; Francisco José B Reifschneider ${ }^{1 ; 2}$; Antônio Williams Moita ${ }^{1}$; Geraldo da S e Souza ${ }^{2}$ \\ ${ }^{1}$ Embrapa Hortaliças, C. Postal 218, 70351-970 Brasília-DF; ${ }^{2}$ Embrapa Sede, Parque Estação Biológica, 70770-901 Brasília-DF; silvia@ \\ cnph.embrapa.br; fjbr@cnph.embrapa.br; moita@cnph.embrapa.br; geraldo.souza@embrapa.Br
}

\section{RESUMO}

Melhoristas de Capsicum da Embrapa Hortaliças estão orientando parte de seus esforços sob a ótica dos consumidores intermediários e finais com objetivo de aumentar as chances de sucesso de longo prazo do desenvolvimento de novas cultivares. Nesse cenário, este trabalho ilustra uma pesquisa, iniciada em 2007, com consumidores de pimentão do Distrito Federal para conhecer as principais características que são levadas em consideração no ato da compra. $\mathrm{O}$ estudo foi dividido em duas partes: um piloto com 444 consumidores de um grande supermercado e um levantamento com 500 consumidores finais de pimentão in natura de cinco supermercados de Brasília. Utilizou-se a análise de dados categóricos por meio do procedimento CATMOD do SAS para verificar a ordenação de importância dos atributos, os contrastes das médias, bem como a influência do perfil do comprador na avaliação das características envolvidas na compra. Em complemento, usou-se a análise de correspondência para a representação gráfica das relações multidimensionais das distâncias do grau de importância dos atributos do pimentão. Os consumidores enfatizaram a textura e as características relacionadas ao aspecto visual durante a compra do pimentão. Em adição, foram detectadas diferenças de percepção de alguns atributos dentre os consumidores quando segmentados por gênero, idade, grau de escolaridade, estado civil e localização do supermercado. Destaca-se, ainda, a maior preocupação das características funcionais por pessoas mais idosas. Estudos dessa natureza são essenciais para melhor orientar os programas de melhoramento genético vegetal.

Palavras-chave: Capsicum annuum, consumo, qualidade, supermercado.

\section{ABSTRACT}

Attributes of vegetables based on consumer's needs: a case study on bell peppers in Distrito Federal, Brazil

Capsicum (chili and bell peppers) breeders at Embrapa Vegetables are focusing project activities to satisfy the demands of both growers and final consumers in order to increase the chances of success in, long-term development of new cultivars. So, a prospection study on consumer demands and perception on bell peppers was started in 2007 in order to identify the main attributes taken in consideration during the act of purchase. This work was divided in two parts: a pilot study with 444 consumers at one supermarket and a survey with 500 consumers of fresh bell pepper, carried out by interviews at five supermarkets in Brasilia, Brazil, which represent distinct income segments. Data were analyzed by categorical data analysis using SAS (Procedure CATMOD) to rank the attributes in order of importance; to contrast the attribute means; and to verify the psychosocial influence on ranking the attributes during the purchase act. In addition, correspondence analysis was used for graphical representation of the attributes separated by the level of importance. During purchase, consumers prioritize the attributes related to the visual aspect (appearance) and firmness of the fruits. Also, differences of perception by gender, age, educational level, marital status and supermarket location were detected. Based on the collected evidence, elderly people are more concerned about nutritional value. Plant breeding programs can be better focused utilizing inputs from similar studies.

Keywords: Capsicum annuum, consumption, quality, supermarket.

(Recebido para publicação em 14 de agosto de 2009; aceito em 26 de fevereiro de 2010)

(Received on August 14, 2009; accepted on February 26, 2010)

$\mathrm{O}$ pimentão está entre as hortaliças mais consumidas no Brasil e ocupa uma área significativa de plantio, concentrada em São Paulo, Santa Catarina, Minas Gerais, Rio de Janeiro e estados do Nordeste (Maldonado, 2000). Henz et al. (2007) enfatizam também a produção do estado do Paraná no cenário do pimentão e o Distrito Federal como um dos principais pólos de produção de pimentão em cultivo protegido no país, tendo área aproximada de 50 ha cultivada anualmente. Dados do Instituto de Economia Agrícola (IEA) revelam que, em 2008, a produção do estado de São Paulo foi de aproximadamente 60.140 toneladas. No Paraná e em Santa
Catarina, as Secretarias de Agricultura informaram que a produção foi 63.600 toneladas em 2003. Em Minas Gerais, em 2008 foram comercializados em torno de 12.000 toneladas de pimentão na Ceasa de Belo Horizonte (Seapa, 2009).

Dada a importância econômica dessa hortaliça, as empresas de sementes têm lançado muitas cultivares no mercado, com grandes variações de formato, tamanho e cor. Esse mercado de sementes movimenta R \$ 8,7 milhões por ano (Della Vecchia, 2007). Atualmente, conta-se com mais de 60 cultivares, com destaque para os híbridos que predominam no cultivo protegido e têm presença significativa em campo aberto (Abcsem, 2007). Entre estes, os híbridos Margarita, Paloma, Rúbia, Escarlata e Magali R são de grande importância no Distrito Federal. No entanto, observase uma rápida e constante substituição de genótipos pelas companhias produtoras de semente, fazendo com que a vida média do híbrido no mercado seja curta. Vale ressaltar que ainda há forte presença de cultivares de polinização aberta de pimentão em campo aberto (Abcsem, 2007) e, pela característica do setor público, há interesse no desenvolvimento deste tipo de genótipo que possa competir com os híbridos que têm alto custo de semente. 
Presentemente, nota-se que os híbridos são obtidos com o objetivo de suprir principalmente os interesses das companhias globalizadas de sementes, assim como as necessidades do produtor, já que os atributos ressaltados são resistência a doenças, alto vigor, produtividade, precocidade de produção e uniformidade. Mesmo em outras espécies, principalmente frutas e hortaliças, os projetos de pesquisa buscam principalmente atender problemas de cultivo da planta. Por outro lado, os atributos-chave para os demais agentes da cadeia, em especial a preferência do consumidor, têm sido pouco considerados como alvos das pesquisas de melhoramento genético (Matsuura et al., 2004).

$\mathrm{Na}$ esfera do consumidor final, pesquisas sobre o seu comportamento frente aos setores de frutas e hortaliças sinalizaram uma preocupação incipiente sobre a captação das necessidades dos diversos agentes da cadeia. Segundo Saabor (2001), a compreensão dos hábitos do consumidor é uma vantagem competitiva na venda de frutas e hortaliças.

Souza (2005) menciona que, na pesquisa realizada pela ABRAS sobre frutas e hortaliças em 1998, foram identificados oito estratos socioeconômicos: "...o valor nutricional, a qualidade e o sabor foram apontados como fundamentais para a decisão de compra...". Em complemento, Kader (2002) pondera que os atributos que mais influenciam na decisão de compra de alimentos frescos são: sabor ou aroma, textura e aparência, uma vez que os consumidores se baseiam no visual e no que aparentam ter gosto bom. Como o cliente não tem acesso à visualização interior do fruto, utiliza-se de sua experiência anterior para avaliar o produto (Andreuccetti et al., 2005). Os mesmos autores reforçam a idéia de que a aparência é fator decisivo no ato da compra, em uma pesquisa realizada com tomate de mesa. Ragaert et al. (2004) enfatizam frescor e sabor e Van der Pol \& Ryan (1996) mencionam que há consumidores pontuando preço e qualidade (frescor, aparência e valor nutricional) como atributos mais importantes de frutas e hortaliças.

Para o pimentão, Frank et al. (2001) relatam que um número de atributos pode afetar a preferência de consumidores americanos, incluindo os relacionados com aspecto (cor, tamanho, formato), valor nutricional (vitamina C, pro-vitamina A, minerais e fibras), intenção de uso (congelado, pré-cortado, fruto inteiro para consumo) e preço. Dentre esses atributos, cor, preço e valor nutricional foram analisados pela técnica de análise conjunta com 435 consumidores e o atributo "cor" foi três vezes mais importante que preço no processo de compra. Todavia, a vitamina $\mathrm{C}$ foi considerada um atributo irrelevante aos consumidores americanos, contrariando a afirmação de Souza (2005) para os consumidores brasileiros.

Estudos à parte destacam características do pimentão relacionados à pós-colheita que são enfatizadas pelos agentes intermediários (atacadistas e varejistas). Esforços do Instituto Brasileiro de Qualidade em Horticultura, vinculado à Ceagesp tiveram como resultado a elaboração de normas de classificação do pimentão quanto a comprimento, diâmetro, cor e defeitos (Gorenstein \& Gutierrez, 2000). Estes estão relacionados com incidência de frutos tortos, murchos e danos fisiológicos, mecânicos e relacionados a doenças e pragas (Lana et al., 1999) que influenciam na aparência e na textura do pimentão, são características ressaltadas pelos consumidores na literatura.

$\mathrm{Na}$ perspectiva de Gorenstein \& Gutierrez (2000), os padrões visam proporcionar uma linguagem comum no comércio entre os agentes da cadeia para se ter uma remuneração de incentivo que valorize os produtos de melhor qualidade. Entretanto, em visitas aos supermercados e centrais de distribuição, os agentes da cadeia seguem só alguns pontos da norma para classificação do produto, haja vista que ela é de adesão voluntária.

Face a esse cenário, pesquisadores do programa de melhoramento de Capsicum (pimentão e pimentas) da Embrapa Hortaliças estão procurando determinar as características de pimentas e pimentões de maior interesse por parte dos consumidores intermediários e finais, dada a importância do agronegócio destas culturas no Brasil. Blackwell et al. (2005) referendam que um produto novo deve satisfazer às necessidades, desejos e expectativas de seus principais consumidores, sejam intermediários e/ ou finais. Pesquisadores como Clark \& Wheelwright (1993), Ciccantelli \& Magidson (1993) e Cooper (1999), dentre outros, mencionam a importância de articular o processo de novos produtos com as necessidades de todos os seus consumidores com objetivo de garantir o sucesso no lançamento desses produtos.

Assim, iniciou-se um estudo de prospecção de demandas da cadeia de Capsicum no intuito de conhecer melhor as searas dos consumidores (intermediários e finais), em congruência com a sinalização por parte da academia em conhecer o comportamento do consumidor de frutas e hortaliças (Van der Pol \& Ryan, 1996; Frank et al., 2001; Fillion \& Kildcast, 2002; Kader, 2002; Ragaert et al., 2004; Andreuccetti et al., 2005; Peres et al., 2008).

Este trabalho teve como objetivo estudar uma das etapas de prospecção, com o foco na cadeia de pimentão. A pesquisa foi realizada primeiramente com 444 consumidores de um grande supermercado do Distrito Federal e posteriormente com 500 consumidores finais de pimentão in natura de cinco supermercados distintos do Distrito Federal para conhecer os principais atributos do pimentão que são levados em consideração no ato da compra.

\section{MATERIAL E MÉTODOS}

Utilizou-se o método survey na pesquisa, com questionário padronizado e estruturado, por meio de entrevistas com os consumidores, para descobrir certos traços e atributos da população estudada (Babbie, 2003). A estratégia selecionada foi de natureza quantitativa, uma vez que o foco foi quantificar quais atributos do pimentão são considerados mais importantes no ato da compra.

A elaboração do questionário foi realizada por meio de uma pesquisa exploratória: entrevistou-se quarenta compradores/consumidores de pimentão de um grande supermercado de Brasília, no final de dezembro de 2007. Perguntou-se quais atributos os consumidores levam em conta no ato da compra do 
pimentão.

Os resultados foram tabulados e agregados e, a partir deles, elaborou-se um questionário fechado com perguntas sobre o grau de importância dos atributos: preço, tamanho, firmeza, cor uniforme, brilho, sem manchas, sem estrias, superfície lisa, não amassado, teor de vitamina, peso e sabor na hora da compra. Os itens foram mensurados na escala Likert com variação de 1 (nenhuma importância) a 5 (muito importante). Além disso, perguntou-se para os atributos tamanho, formato e peso a preferência de seus tipos aos respondentes que pontuaram nota acima de 3 .

$\mathrm{Na}$ etapa seguinte, no período de janeiro a fevereiro de 2008, às terças, quartas, quintas e sábados, realizou-se a pesquisa piloto com 444 consumidores de um grande supermercado de Brasília. As entrevistas estruturadas da primeira pesquisa ocorreram na seção de hortifrutigranjeiros, em que foram abordados consumidores de diferentes faixas etárias, grau de escolaridade, sexo e estado civil.

Por último, realizou-se uma segunda pesquisa com 500 consumidores de pimentão que abrangesse os consumidores do Distrito Federal. Sendo assim, a pesquisa envolveu cinco supermercados localizados em cinco localidades do Distrito Federal: Ceilândia, Taguatinga, Asa Norte, Riacho Fundo e Recanto das Emas. O supermercado (A) é pequeno e situa-se em Ceilândia. O supermercado (B), em Taguatinga, pertencente a uma rede de supermercados, atende quatro estados e o Distrito Federal, contabilizando 25 lojas. O hipermercado (C), localizado na Asa Norte, pertence a uma rede com 80 lojas em 12 estados e no Distrito Federal. O supermercado (D), em Riacho Fundo, é uma das quatro lojas de um grupo local, que abrange também Samambaia e Taguatinga; e o supermercado (E), localizado no Recanto das Emas, advém de uma rede do Distrito Federal que possui 13 lojas em Ceilândia, Taguatinga, Samambaia, Riacho Fundo e Recanto das Emas.

A escolha desses locais foi baseada nos dados sobre estratificação das regiões administrativas do Distrito Federal em cinco grupos por classe de renda (Codeplan, 1997): a população da Asa
Norte está inserida no primeiro grupo de renda, seguida por Taguatinga, Riacho Fundo, Ceilândia e Recanto das Emas.

Nos meses de novembro de 2008 a fevereiro de 2009 as entrevistas também foram realizadas às terças, quartas, quintas e sábados. Para o cálculo do tamanho das amostras dos dois casos, o intervalo de confiança foi de 95\%. O tamanho da amostra foi calculado a partir de uma amostra piloto, com a utilização dos preceitos de Matar (1997).

O tratamento descritivo dos dados envolveu a preparação deles, cálculo das médias, dos desvios-padrões e distribuição do grau de importância dos atributos. Para verificar a ordenação de importância dos atributos, os contrastes das médias, bem como a influência do perfil do comprador na avaliação dos atributos envolvidos na compra, utilizou-se o procedimento CATMOD do SAS (Stokes et al., 2000), com estudo das características por meio de análise de dados categóricos. De acordo com os autores, dados categóricos resultam de observações de variáveis aleatórias (atributos) cujos valores possíveis são classificados em um conjunto finito de classes ou categorias disjuntas. Exemplos típicos são fornecidos por atributos tais como grau de infestação de uma cultura (forte, médio ou fraco), cor dos olhos e nacionalidade. Tipicamente temse interesse no estudo da distribuição de freqüências de ocorrência das possíveis categorias que são apresentadas em forma de tabelas conhecidas como tabelas de contingência. Estas podem ser uni ou multidimensionais dependendo da dimensão do vetor de atributos de interesse.

Vale ressaltar que a análise de dados categóricos diz respeito à análise das freqüências representadas por uma tabela de contingência. Neste contexto procura-se modelar estatisticamente uma função de interesse das freqüências relativas ou probabilidades empíricas de ocorrência das várias categorias envolvidas. As regressões logísticas para respostas binárias enquadram-se nesta ordem de idéias.

Em adição, usou-se a análise de correspondência para a representação gráfica das relações multidimensionais das distâncias do grau de importância dos atributos do pimentão. A análise de correspondência é uma técnica estatística para representar em um gráfico bi-dimensional as linhas e colunas de uma tabela de contigência. Essa metodologia foi utilizada porque todas as variáveis tomadas são qualitativas (não paramétricas), impossibilitando então um estudo quantitativo que explorasse as relações entre as variáveis (Hair et al., 1998).

\section{RESULTADOS E DISCUSSÃO}

Os resultados obtidos foram agregados em dois tópicos: o primeiro descreve sucintamente o perfil dos consumidores estudados (Tabela 1). O segundo identifica a percepção dos entrevistados na escolha dos atributos que irão influenciar o processo de compra do pimentão.

Observou-se a predominância feminina na compra de pimentão e alguns respondentes masculinos mencionaram que compram hortaliças de vez em quando, pois suas parceiras são as que efetuam as compras. Os dados convergem com as pesquisas realizadas com consumidores de hortaliças minimamente processadas e de tomate por Ragaert et al. (2004), Andreuccetti et al. (2005) e Peres et al. (2008), ao indicar que a mulher ainda é a maior responsável pelas compras de alimentação da família.

$\mathrm{Na}$ pesquisa piloto, a idade dos entrevistados concentrou-se entre 31 e 50 anos $(56 \%)$. Na segunda, a idade dos entrevistados apresentou maior porcentagem nas seguintes categorias: até 30 anos com $21,5 \%$ e entre 50 e 60 com $25,4 \%$. Nas duas pesquisas, mais de $50 \%$ das pessoas entrevistadas possuem mais de 30 anos, inferindo que o perfil de compradores de pimentão é de pessoas com maior experiência como consumidores.

Em adição, as pessoas casadas corresponderam na primeira pesquisa a $72,3 \%$ e na segunda, $43,6 \%$. Isso sugere que uma parte considerável dos compradores de pimentão dos supermercados está numa faixa etária entre 30 e 60 anos, são casados e possivelmente têm o hábito de cozinhar em casa.

O grau de escolaridade diferiu entre as duas pesquisas: na primeira prevaleceram indivíduos com nível su- 
Tabela 1. Perfil dos entrevistados quanto ao gênero, idade, estado civil e grau de escolaridade (profile of respondents by gender, age, maritus status and scholar degree). Brasília, Embrapa Hortaliças, 2009.

\begin{tabular}{lcc}
\hline Perfil dos entrevistados & $\mathbf{2 0 0 7 - 2 0 0 8}$ & $\mathbf{2 0 0 8 - 2 0 0 9}$ \\
\hline Sexo & & \\
\hline Feminino & $72,8 \%$ & $62,9 \%$ \\
Masculino & $27,2 \%$ & $37,1 \%$ \\
\hline Idade & & \\
\hline Até 30 anos & $14,8 \%$ & $21,3 \%$ \\
$31-40$ anos & $27,6 \%$ & $15,4 \%$ \\
$41-50$ anos & $28,4 \%$ & $19,1 \%$ \\
$51-60$ anos & $17,1 \%$ & $25,4 \%$ \\
Acima de 60 anos & $12,1 \%$ & $18,7 \%$ \\
\hline Estado civil & & $32,9 \%$ \\
Solteiro & $16,4 \%$ & $43,0 \%$ \\
Casado & $72,6 \%$ & $12,4 \%$ \\
Divorciado & $8,0 \%$ & $11,7 \%$ \\
Outros & $3,0 \%$ & \\
\hline Grau de escolaridade & & $19,3 \%$ \\
\hline Primeiro grau incompleto & $2,3 \%$ & $17,6 \%$ \\
Primeiro grau completo & $6,8 \%$ & $43,6 \%$ \\
Segundo grau completo & $35,1 \%$ & $19,5 \%$ \\
Terceiro grau completo & $55,8 \%$ &
\end{tabular}

perior $(55,8 \%)$; na segunda, as pessoas que possuem segundo grau completo (43,6\%). Não foi perguntado o nível de renda dos entrevistados, já que esse dado pode ser parcialmente inferido pela
\& Kanuk (2000), quanto maior o grau de instrução de um indivíduo, maior a probabilidade de ter uma renda maior, uma posição social admirada e respeitada. Justifica-se a alta concentração de pessoas com terceiro grau na primeira amostra pela localização do supermercado em uma região de classe média.

A segunda parte da pesquisa foi pautada na identificação das principais características do pimentão. Para ordenar os dados quanto ao grau de importância, utilizou-se procedimento CATMOD do SAS, mediante a técnica de regressão múltipla e distribuição não normal (Tabela 2). Alguns contrastes entre os atributos apresentaram diferenças não significativas ao nível de 5\%.

Verificou-se poucas diferenças na ordenação dos atributos das duas pesquisas. Oito características (entre as treze perguntadas) obtiveram notas acima de quatro (importante). Preço e valor nutricional apresentaram médias acima de 3,4 (entre alguma importância a importante). Na primeira pesquisa, somente peso teve nota abaixo de 3 , enquanto que na segunda ocorreu com as características tamanho, formato e peso.

Os desvios-padrões dos atributos que tiveram as menores médias foram os mais elevados, conforme Tabela 2 .

Tabela 2. Ordenação de atributos do pimentão utilizando a técnica do CATMOD (ranking of bell pepper's attributes using the CATMOD technique). Brasília, Embrapa Hortaliças, 2009.

\begin{tabular}{|c|c|c|c|c|c|c|c|}
\hline \multicolumn{4}{|c|}{ Pesquisa 2007-2008 } & \multicolumn{4}{|c|}{ Pesquisa 2008-2009 } \\
\hline Atributos & & Iédia & Desvio padrão & Atributo & Médi: & & Desvio padrão \\
\hline Não amassado & $4,86 \mathrm{a}$ & & 0,40 & Não amassado & $4,76 \mathrm{a}$ & & 0,54 \\
\hline Firmeza & $4,76 \mathrm{~b}$ & & 0,46 & Sem manchas & $4,62 t$ & & 0,69 \\
\hline Sem manchas & 4,62 & $\mathrm{c}$ & 0,61 & Firmeza & 4,53 & & 0,76 \\
\hline Sabor do pimentão & 4,41 & $\mathrm{~d}$ & 0,89 & Superfície lisa & 4,48 & $\mathrm{~cd}$ & 0,90 \\
\hline Superfície lisa & 4,39 & $\mathrm{~d}$ & 0,93 & Sem estrias & 4,38 & $\mathrm{~d}$ & 1,05 \\
\hline Sem estrias & 4,22 & e & 1,16 & Sabor do pimentão & 4,12 & $\mathrm{e}$ & 1,10 \\
\hline Cor uniforme & 4,15 & $\mathrm{f}$ & 1,15 & Cor uniforme & 4,08 & $\mathrm{e}$ & 1,21 \\
\hline Brilho & 4,05 & fg & 1,17 & Brilho & 4,07 & $\mathrm{e}$ & 1,25 \\
\hline Preço & 3,67 & g & 1,34 & Preço & 3,88 & $\mathrm{f}$ & 1,47 \\
\hline Valor Nutricional & 3,42 & $\mathrm{~h}$ & 1,69 & Valor Nutricional & 3,74 & g & 1,52 \\
\hline Tamanho & 3,27 & $\mathrm{~h}$ & 1,20 & Tamanho & 2,96 & $\mathrm{i}$ & 1,61 \\
\hline Formato & 3,05 & $\mathrm{i}$ & 1,46 & Formato & 2,88 & $\mathrm{i}$ & 1,56 \\
\hline Peso & 2,64 & $\mathrm{j}$ & 1,56 & Peso & 2,63 & $\mathrm{i}$ & 1,81 \\
\hline
\end{tabular}

*Médias seguidas de letras diferentes na coluna diferiram significativamente a $5 \%$ de probabilidade de erro (means with different letters on the collumn differ significantly to $5 \%$ of error probability). 
Observou-se que as notas de importância foram polarizadas nos extremos "nenhuma importância" e "importante/ muito importante". Para as demais, os desvios-padrões não excederam 1,45, com pouca dispersão dos dados.

Efetuou-se análise de correspondência para uma melhor visualização da disposição dos atributos quanto à importância, com variância no eixo x (dimensão 1) de 78,8\% (pesquisa piloto) e $90,9 \%$ (segunda pesquisa), ilustradas

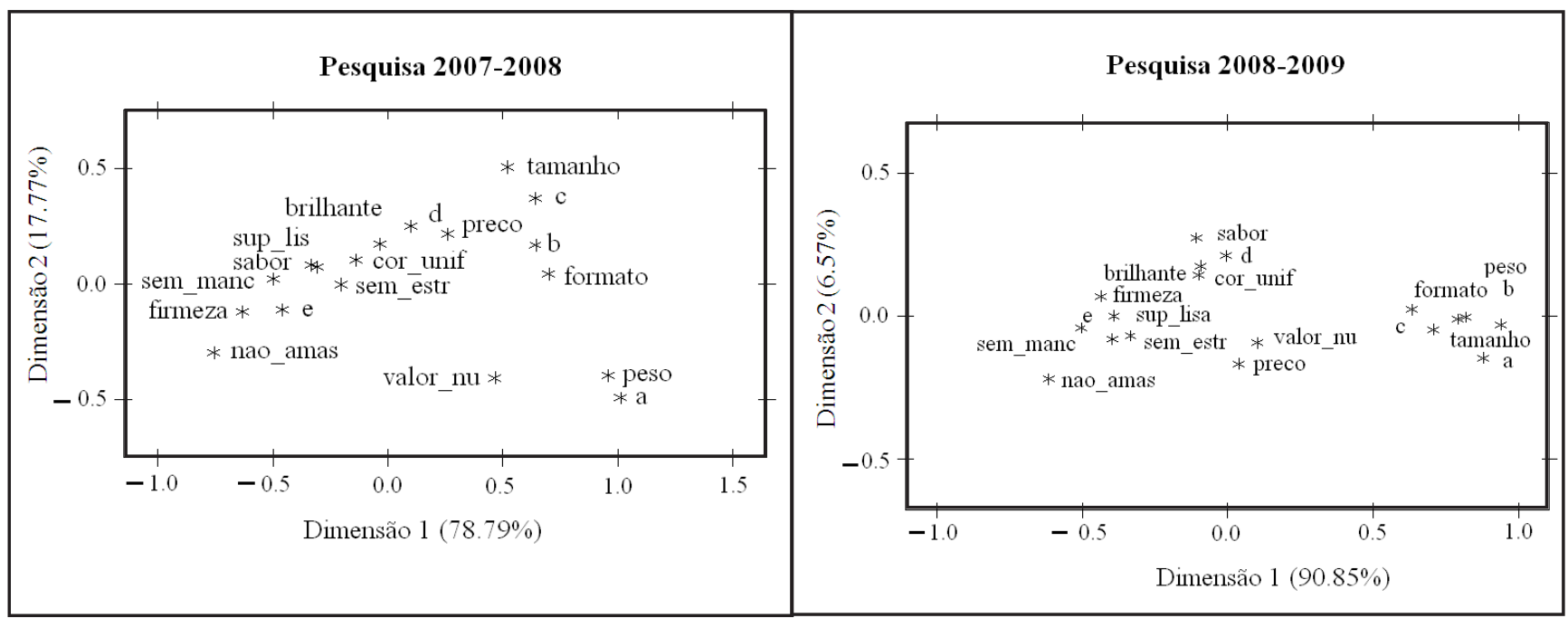

Figura 1. Distribuição espacial dos atributos de pimentão em função da importância (spatial distribution of Bell pepper attributes based on importance). Brasília, Embrapa Hortaliças, 2009.

Legenda: $\mathrm{e}=$ muito importante; $\mathrm{d}=$ importante; $\mathrm{c}=$ alguma importância; $\mathrm{b}=$ pouca importância; $\mathrm{a}=$ nenhuma importância (e=very important; $\mathrm{d}=$ important; $\mathrm{c}=$ slightly important; $\mathrm{b}=$ less important; $\mathrm{a}=$ unimportant).

Tabela 3a. Importância dos atributos segundo o perfil dos entrevistados da pesquisa de 2007/2008 (importance of attributes according to the profile of survey respondents 2007/2008). Brasília, Embrapa Hortaliças, 2009.

\begin{tabular}{|c|c|c|c|c|c|c|c|c|}
\hline & $\begin{array}{c}\text { Não } \\
\text { amassado }\end{array}$ & Firmeza & $\begin{array}{c}\text { Sem } \\
\text { manchas }\end{array}$ & $\begin{array}{l}\text { Sabor do } \\
\text { pimentão }\end{array}$ & $\begin{array}{c}\text { Superfície } \\
\text { lisa }\end{array}$ & $\begin{array}{c}\text { Sem } \\
\text { estrias }\end{array}$ & $\begin{array}{c}\text { Cor } \\
\text { uniforme }\end{array}$ & Brilho \\
\hline Sexo & $\mathrm{p}=0,43$ & $\mathrm{p}=0,02$ & $\mathrm{p}=0,28$ & $\mathrm{p}=0,38$ & $\mathrm{p}=0,009$ & $\mathrm{p}<0,001$ & $\mathrm{p}=0,83$ & $\mathrm{p}=0,01$ \\
\hline Masculino & $4,84 a^{*}$ & $4,67 \mathrm{a}$ & $4,56 \mathrm{a}$ & $4,35 \mathrm{a}$ & $4,19 \mathrm{a}$ & $3,79 \mathrm{a}$ & $4,13 \mathrm{a}$ & $3,81 \mathrm{a}$ \\
\hline Feminino & $4,88 \mathrm{a}$ & $4,79 \mathrm{~b}$ & $4,64 \mathrm{a}$ & $4,44 \mathrm{a}$ & $4,46 \mathrm{~b}$ & $4,38 \mathrm{~b}$ & $4,16 \mathrm{a}$ & $4,14 b$ \\
\hline Grau de escolaridade & $\mathrm{p}=0,07$ & $\mathrm{p}=0,53$ & $\mathrm{p}=0,29$ & $\mathrm{p}=0,69$ & $\mathrm{p}=0,15$ & $\mathrm{p}=0,73$ & $\mathrm{p}=0,79$ & $\mathrm{p}=0,70$ \\
\hline Primeiro grau incompleto & $4,43 \mathrm{ab}$ & $4,42 \mathrm{a}$ & $4,57 \mathrm{a}$ & $4,29 a$ & $3,14 \mathrm{a}$ & $4,00 \mathrm{a}$ & $4,29 a$ & $3,57 \mathrm{a}$ \\
\hline Primeiro grau completo & $4,95 \mathrm{a}$ & $4,77 \mathrm{a}$ & $4,77 \mathrm{a}$ & $4,27 \mathrm{a}$ & $4,50 \mathrm{a}$ & $3,95 \mathrm{a}$ & $4,09 \mathrm{a}$ & $4,18 \mathrm{a}$ \\
\hline Segundo grau completo & $4,82 b^{* *}$ & $4,78 \mathrm{a}$ & $4,65 \mathrm{a}$ & $4,48 \mathrm{a}$ & $4,34 \mathrm{a}$ & $4,27 \mathrm{a}$ & $4,22 \mathrm{a}$ & $4,14 \mathrm{a}$ \\
\hline Terceiro grau completo & $4,89 \mathrm{ab}$ & $4,74 \mathrm{a}$ & $4,59 \mathrm{a}$ & $4,43 \mathrm{a}$ & $4,48 \mathrm{a}$ & $4,19 \mathrm{a}$ & $4,10 \mathrm{a}$ & $4,05 \mathrm{a}$ \\
\hline Idade & $\mathrm{p}=0,63$ & $\mathrm{p}=0,34$ & $\mathrm{p}=0,95$ & $\mathrm{p}=0,68$ & $\mathrm{p}=0,19$ & $\mathrm{p}=0,33$ & $\mathrm{p}=0,02$ & $\mathrm{p}=0,50$ \\
\hline Até 30 anos & $4,82 \mathrm{a}$ & $4,71 \mathrm{a}$ & $4,68 \mathrm{a}$ & $4,52 \mathrm{a}$ & $4,51 \mathrm{a}$ & $4,38 \mathrm{a}$ & $4,41 \mathrm{a}$ & $4,15 \mathrm{a}$ \\
\hline $31-40$ anos & $4,89 \mathrm{a}$ & $4,74 \mathrm{a}$ & $4,61 \mathrm{a}$ & $4,47 \mathrm{a}$ & $4,50 \mathrm{a}$ & $4,24 \mathrm{a}$ & $4,10 b^{* * *}$ & $4,14 \mathrm{a}$ \\
\hline $41-50$ anos & $4,88 \mathrm{a}$ & $4,82 \mathrm{a}$ & $4,62 \mathrm{a}$ & $4,38 \mathrm{a}$ & $4,35 \mathrm{a}$ & $4,23 \mathrm{a}$ & $4,28 \mathrm{a}$ & $4,04 \mathrm{a}$ \\
\hline $51-60$ anos & $4,91 \mathrm{a}$ & $4,73 \mathrm{a}$ & $4,63 \mathrm{a}$ & $4,39 a$ & $4,31 \mathrm{a}$ & $4,21 \mathrm{a}$ & $3,83 \mathrm{~b}$ & $3,95 \mathrm{a}$ \\
\hline Mais que 60 anos & $4,83 \mathrm{a}$ & $4,79 \mathrm{a}$ & $4,60 \mathrm{a}$ & $4,45 \mathrm{a}$ & $4,19 \mathrm{a}$ & $3,89 \mathrm{a}$ & $4,04 \mathrm{ab}$ & $3,83 \mathrm{a}$ \\
\hline Estado civil & $\mathrm{p}=0,34$ & $\mathrm{p}=0,60$ & $\mathrm{p}=0,76$ & $\mathrm{p}=0,39$ & $\mathrm{p}=0,86$ & $\mathrm{p}=0,31$ & $\mathrm{p}=0,16$ & $\mathrm{p}=0,81$ \\
\hline Solteiro & $4,82 \mathrm{a}$ & $4,78 \mathrm{a}$ & $4,64 \mathrm{a}$ & $4,50 \mathrm{a}$ & $4,39 a$ & $4,32 \mathrm{a}$ & $3,96 a$ & $4,11 \mathrm{a}$ \\
\hline Casado & $4,88 \mathrm{a}$ & $4,75 \mathrm{a}$ & $4,62 \mathrm{a}$ & $4,42 \mathrm{a}$ & $4,41 \mathrm{a}$ & $4,18 \mathrm{a}$ & $4,19 \mathrm{a}$ & $4,08 \mathrm{a}$ \\
\hline
\end{tabular}

*Médias seguidas de letras diferentes na coluna diferiram significativamente a $5 \%$ de probabilidade de erro; $* * 2-3$ foi significativa porque as estimativas do desvio padrão foram maiores do que 1-4; ***1-2 e 2-3 foram significativas porque as estimativas do desvio padrão foram maiores do que $1-5$ e $3-5$. (means with different letters on the collumn differ significantly to $5 \%$ of error probability; **2-3 was significant because the estimates of standard deviation were higher than 1-4;***1-2 and 2-3 were significant because the estimates of standard deviation were higher than 1-5 and 3-5). 
Tabela 3a. (continuação ...) Importância dos atributos segundo o perfil dos entrevistados da pesquisa de 2007/2008 (continuation ... importance of attributes according to the profile of survey respondents 2007/2008) . Brasília, Embrapa Hortaliças, 2009.

\begin{tabular}{llllll}
\hline & Preço & $\begin{array}{l}\text { Valor } \\
\text { nutricional }\end{array}$ & Tamanho & Formato & Peso \\
\hline Sexo & $\mathrm{p}=0,19$ & $\mathrm{p}=0,77$ & $\mathrm{p}=0,88$ & $\mathrm{p}=0,13$ & $\mathrm{p}=0,92$ \\
\hline Masculino & $3,53 \mathrm{a}$ & $3,22 \mathrm{a}$ & $3,41 \mathrm{a}$ & $3,22 \mathrm{a}$ & $2,62 \mathrm{a}$ \\
Feminino & $3,72 \mathrm{a}$ & $3,27 \mathrm{a}$ & $3,43 \mathrm{a}$ & $2,99 \mathrm{a}$ & $2,64 \mathrm{a}$ \\
\hline Grau de escolaridade & $\mathrm{p}=0,28$ & $\mathrm{P}<0,0001$ & $\mathrm{p}=0,0008$ & $\mathrm{p}=0,03$ & $\mathrm{p}=0,29$ \\
\hline Primeiro grau incompleto & $4,00 \mathrm{a}$ & $1,43 \mathrm{~b}$ & $4,28 \mathrm{a}$ & $2,71 \mathrm{ab}$ & $3,14 \mathrm{a}$ \\
Primeiro grau completo & $3,72 \mathrm{a}$ & $3,50 \mathrm{a}$ & $3,04 \mathrm{~b}$ & $2,18 \mathrm{~b}$ & $2,50 \mathrm{a}$ \\
Segundo grau completo & $3,78 \mathrm{a}$ & $3,41 \mathrm{a}$ & $3,60 \mathrm{~b}$ & $3,11 \mathrm{a}$ & $2,78 \mathrm{a}$ \\
Terceiro grau completo & $3,52 \mathrm{a}$ & $3,21 \mathrm{a}$ & $3,31 \mathrm{~b}$ & $3,08 \mathrm{a}$ & $2,49 \mathrm{a}$ \\
\hline Idade & $\mathrm{p}=0,09$ & $\mathrm{p}=0,04$ & $\mathrm{p}=0,51$ & $\mathrm{p}=0,24$ & $\mathrm{p}=0,51$ \\
\hline Até 30 anos & $3,65 \mathrm{ab}$ & $3,11 \mathrm{~b}$ & $3,58 \mathrm{a}$ & $2,85 \mathrm{a}$ & $2,63 \mathrm{a}$ \\
31-40 anos & $3,69 \mathrm{ab}$ & $2,97 \mathrm{~b}$ & $3,54 \mathrm{a}$ & $3,24 \mathrm{a}$ & $2,45 \mathrm{a}$ \\
41-50 anos & $3,42 \mathrm{~b}$ & $3,36 \mathrm{ab}$ & $3,35 \mathrm{a}$ & $3,16 \mathrm{a}$ & $2,74 \mathrm{a}$ \\
51-60 anos & $3,77 \mathrm{ab}$ & $3,67 \mathrm{ab}$ & $3,39 \mathrm{a}$ & $2,88 \mathrm{a}$ & $2,59 \mathrm{a}$ \\
Mais que 60 anos & $4,00 \mathrm{a}$ & $3,49 \mathrm{a}$ & $3,30 \mathrm{a}$ & $2,94 \mathrm{a}$ & $2,81 \mathrm{a}$ \\
\hline Estado civil & $\mathrm{p}=0,36$ & $\mathrm{p}=0,58$ & $\mathrm{p}=0,05$ & $\mathrm{p}=0,04$ & $\mathrm{p}=0,97$ \\
\hline Solteiro & $3,76 \mathrm{a}$ & $3,17 \mathrm{a}$ & $3,64 \mathrm{a}$ & $2,75 \mathrm{a}$ & $2,60 \mathrm{a}$ \\
Casado & $3,60 \mathrm{a}$ & $3,29 \mathrm{a}$ & $3,36 \mathrm{a}$ & $3,10 \mathrm{~b}$ & $2,61 \mathrm{a}$ \\
\hline
\end{tabular}

*Médias seguidas de letras diferentes na coluna diferiram significativamente a $5 \%$ de probabilidade de erro; **2-3 foi significativa porque as estimativas do desvio padrão foram maiores do que 1-4; ***1-2 e 2-3 foram significativas porque as estimativas do desvio padrão foram maiores do que 1-5 e 3-5 (means with different letters on the collumn differ significantly to $5 \%$ of error probability; $* * 2-3$ was significant because the estimates of standard deviation were higher than 1-4; ***1-2 and 2-3 were significant because the estimates of standard deviation were higher than 1-5 and 3-5).

nas Figuras 1 e 2. Pelos dados das variâncias, a leitura da ordenação espacial das características quanto à importância seguiu o eixo X.

Esses resultados indicam que os consumidores priorizam os atributos relacionados à textura, pois gostam do pimentão não amassado e firme. Fillion $\&$ Kildcast (2002) pontuam que a textura é qualidade desejável em frutas e hortaliças, pois está relacionada com frescor. Além disso, os consumidores destacaram os atributos relacionados ao aspecto visual do pimentão, já que eles selecionam os pimentões que não estejam manchados, sem estrias, lisos, com coloração uniforme e brilhantes. Essas características são enfatizadas tanto pelos consumidores quanto pela norma de classificação do pimentão. Destaca-se também a importância do sabor, mesmo sem experimentá-lo no ato da compra. Aparentemente, os consumidores utilizam-se de experiências e formato, todavia, merecem atenção por apresentarem médias próximas à tipologia de alguma importância e os desvios-padrões tiveram valores acima de 1,45. Entre os entrevistados, 355 respondentes na primeira pesquisa e 306 respondentes na segunda relataram que o tamanho varia de alguma importância a muito importante e desses $24,8 \%$ e $35,9 \%$ preferem os pequenos e 63,1 e $43,8 \%$ os médios, respectivamente. Sobre o formato, 257 pessoas no primeiro estudo e 282 no segundo responderam que a característica tem mais que alguma importância e dentre eles 41,6\% e 47,2\% preferem os cônicos enquanto que $58,5 \%$ e $40,8 \%$ escolhem o pimentão de formato bloco respectivamente.

Mesmo assim, observou-se que os pimentões dispostos no mercado apresentam um padrão de tamanho de médio para grande e formato de bloco e que a presença do formato cônico está diminuindo nas prateleiras dos supermercados, pelo menos sazonalmente. Dessa forma, pode-se deduzir que os consumidores não foram consultados na definição do padrão do pimentão, ressaltando a afirmação feita por Matsuura et al. (2004), de que os projetos de pesquisa atentam principalmente para os problemas de cultivo da planta e que os atributos-chave para os clientes finais têm sido pouco considerados como alvos das pesquisas de melhoramento genético.

A pesquisa também avaliou as diferenças de percepção dos perfis dos consumidores com relação aos atributos, ilustradas nas Tabelas 3a e 3b. É fundamental identificar as variáveis demográficas que estão relacionadas como a propensão a consumir determinados produtos para definir um posicionamento adequado no mercado. No âmbito do gênero, em algumas sociedades este pode ainda ser determinador no papel de compra (Sheth et al., 2001).

Pelos resultados, foram verificadas diferenças significativas entre homens e mulheres em relação à importância de algumas características nas duas pesquisas. Na primeira, as mulheres atribuíram maior importância para firmeza e atributos relacionados à superfície do pimentão. Na segunda, maior importância foi atribuída à característica firmeza 
Tabela 3b. Importância dos atributos segundo o perfil dos entrevistados da pesquisa de 2008/2009 (importance of attributes according to the profile of survey respondents 2007/2008). Brasília, Embrapa Hortaliças, 2009.

\begin{tabular}{|c|c|c|c|c|c|c|c|c|}
\hline & $\begin{array}{c}\text { Não } \\
\text { amassado }\end{array}$ & $\begin{array}{c}\text { Sem } \\
\text { mancha }\end{array}$ & Firmeza & $\begin{array}{c}\text { Superfície } \\
\text { lisa }\end{array}$ & Sem estria & Sabor & $\begin{array}{c}\text { Cor } \\
\text { uniforme } \\
\end{array}$ & Brilhante \\
\hline Sexo & $\mathrm{p}=0,40$ & $\mathrm{p}=0,41$ & $\mathrm{p}=0,023$ & $\mathrm{p}=0,6$ & $\mathrm{p}=0,18$ & $\mathrm{p}=0,37$ & $\mathrm{p}=0,54$ & $\mathrm{p}=0,78$ \\
\hline Masculino & $4,73 a^{*}$ & $4,58 \mathrm{a}$ & $4,41 \mathrm{a}$ & $4,45 \mathrm{a}$ & $4,29 a$ & $4,06 \mathrm{a}$ & $4,03 \mathrm{a}$ & $4,09 a$ \\
\hline Feminino & $4,77 \mathrm{a}$ & $4,64 \mathrm{a}$ & $4,59 \mathrm{~b}$ & $4,49 \mathrm{a}$ & $4,43 \mathrm{a}$ & $4,15 \mathrm{a}$ & $4,10 \mathrm{a}$ & $4,05 \mathrm{a}$ \\
\hline Grau de escolaridade & $\mathrm{p}=0,23$ & $\mathrm{p}=0,55$ & $\mathrm{p}=0,41$ & $\mathrm{p}=0,71$ & $\mathrm{p}=0,33$ & $\mathrm{p}=0,59$ & $\mathrm{p}=0,29$ & $\mathrm{p}=0,08$ \\
\hline Primeiro grau incompleto & $4,74 a$ & $4,70 \mathrm{a}$ & $4,43 a$ & $4,42 \mathrm{a}$ & $4,18 \mathrm{a}$ & $3,97 \mathrm{a}$ & $3,94 \mathrm{a}$ & $3,74 b$ \\
\hline Primeiro grau completo & $4,71 \mathrm{a}$ & $4,60 \mathrm{a}$ & $4,49 \mathrm{a}$ & $4,41 \mathrm{a}$ & $4,51 \mathrm{a}$ & $4,20 \mathrm{a}$ & $4,24 \mathrm{a}$ & $4,09 \mathrm{ab}$ \\
\hline Segundo grau completo & $4,74 \mathrm{a}$ & $4,59 \mathrm{a}$ & $4,53 \mathrm{a}$ & $4,40 \mathrm{a}$ & $4,41 \mathrm{a}$ & $4,15 \mathrm{a}$ & $4,12 \mathrm{a}$ & $4,20 \mathrm{a}$ \\
\hline Terceiro grau completo & $4,85 \mathrm{a}$ & $4,61 \mathrm{a}$ & $4,63 \mathrm{a}$ & $4,55 \mathrm{a}$ & $4,41 \mathrm{a}$ & $4,11 \mathrm{a}$ & $3,97 \mathrm{a}$ & $4,13 \mathrm{ab}$ \\
\hline Idade & $\mathrm{p}=0,47$ & $\mathrm{p}=0,34$ & $\mathrm{p}=0,75$ & $\mathrm{p}=0,74$ & $\mathrm{p}=0,04$ & $\mathrm{p}=0,98$ & $\mathrm{p}=0,67$ & $\mathrm{p}=0,57$ \\
\hline $31-40$ anos & $4,70 \mathrm{a}$ & $4,52 \mathrm{a}$ & $4,44 a$ & $4,46 a$ & $4,42 \mathrm{ab}$ & $4,06 \mathrm{a}$ & $3,90 \mathrm{a}$ & $3,96 a$ \\
\hline $41-50$ anos & $4,83 \mathrm{a}$ & $4,66 a$ & $4,57 \mathrm{a}$ & $4,49 \mathrm{a}$ & $4,22 b$ & $4,16 \mathrm{a}$ & $4,12 \mathrm{a}$ & $4,23 \mathrm{a}$ \\
\hline $51-60$ anos & $4,76 a$ & $4,72 \mathrm{a}$ & $4,59 \mathrm{a}$ & $4,49 \mathrm{a}$ & $4,37 \mathrm{ab}$ & $4,13 a$ & $4,09 \mathrm{a}$ & $4,10 \mathrm{a}$ \\
\hline Mais que 60 anos & $4,77 \mathrm{a}$ & $4,58 \mathrm{a}$ & $4,54 \mathrm{a}$ & $4,54 \mathrm{a}$ & $4,57 \mathrm{a}$ & $4,11 \mathrm{a}$ & $4,15 \mathrm{a}$ & $4,10 \mathrm{a}$ \\
\hline $31-40$ anos & $4,75 \mathrm{a}$ & $4,64 \mathrm{a}$ & $4,49 \mathrm{a}$ & $4,37 \mathrm{a}$ & $4,19 \mathrm{~b}$ & $4,14 \mathrm{a}$ & $4,11 \mathrm{a}$ & $3,98 \mathrm{a}$ \\
\hline Estado civil & $\mathrm{p}=0,38$ & $\mathrm{p}=0,41$ & $\mathrm{p}=0,71$ & $\mathrm{p}=0,45$ & $\mathrm{p}=0,49$ & $\mathrm{p}=0,31$ & $\mathrm{p}=0,88$ & $\mathrm{p}=0,98$ \\
\hline Solteiro & $4,71 \mathrm{a}$ & $4,53 a$ & $4,51 \mathrm{a}$ & $4,51 \mathrm{a}$ & $4,37 \mathrm{a}$ & $4,14 \mathrm{a}$ & $4,04 \mathrm{a}$ & $4,04 \mathrm{a}$ \\
\hline Casado & $4,76 \mathrm{a}$ & $4,59 \mathrm{a}$ & $4,48 \mathrm{a}$ & $4,44 \mathrm{a}$ & $4,29 \mathrm{a}$ & $4,02 \mathrm{a}$ & $4,02 \mathrm{a}$ & $4,04 \mathrm{a}$ \\
\hline Local & $\mathrm{p}=0,78$ & $\mathrm{p}=0,008$ & $\mathrm{p}=0,35$ & $\mathrm{p}<0,0001$ & $\mathrm{p}<0,0001$ & $\mathrm{p}=0,76$ & $\mathrm{p}=0,13$ & $\mathrm{p}=0,004$ \\
\hline Ceilândia & $4,77 \mathrm{a}$ & $4,75 a$ & $4,63 a$ & $4,46 b$ & $4,50 \mathrm{ab}$ & $4,07 \mathrm{a}$ & $4,09 \mathrm{ab}$ & $4,05 \mathrm{a}$ \\
\hline Taguatinga & $4,79 \mathrm{a}$ & $4,75 \mathrm{a}$ & $4,57 \mathrm{a}$ & $4,76 \mathrm{a}$ & $4,73 \mathrm{a}$ & $4,17 \mathrm{a}$ & $4,29 a$ & $4,35 \mathrm{a}$ \\
\hline Asa Norte & $4,75 \mathrm{a}$ & $4,56 \mathrm{ab}$ & $4,51 \mathrm{a}$ & $4,50 \mathrm{~b}$ & $4,45 b$ & $4,10 \mathrm{a}$ & $3,90 \mathrm{a}$ & $4,20 \mathrm{a}$ \\
\hline Riacho Fundo & $4,78 \mathrm{a}$ & $4,57 \mathrm{ab}$ & $4,55 \mathrm{a}$ & $4,30 \mathrm{~b}$ & $3,86 \mathrm{c}$ & $4,04 \mathrm{a}$ & $3,95 b$ & $3,71 b$ \\
\hline Recanto das Emas & $4,71 \mathrm{a}$ & $4,48 b$ & $4,38 \mathrm{a}$ & $4,38 \mathrm{~b}$ & $4,38 \mathrm{~b}$ & $4,21 \mathrm{a}$ & $4,19 \mathrm{ab}$ & $4,04 \mathrm{a}$ \\
\hline
\end{tabular}

*Médias seguidas de letras diferentes na coluna diferiram significativamente a 5\% de probabilidade de erro (means with different letters on the collumn differ significantly to $5 \%$ of error probability).

pelas mulheres, enquanto que os homens mostraram maior preocupação com o tamanho e o formato.

Quanto ao grau de escolaridade, tanto na primeira quanto na segunda pesquisa, as pessoas com maior instrução formal deram maior destaque ao valor nutricional do pimentão. Cabe salientar também que preço, tamanho e peso têm importância maior para os entrevistados com primeiro grau. Para as demais características, o grau de escolaridade não interferiu. Como Schiffman \& Kanuk (2000) pressupõem uma associação entre renda e escolaridade, pode-se deduzir que os entrevistados com primeiro grau naturalmente têm uma preocupação maior com preço.

Ao analisar as características sob a ótica da localização dos supermercados, não se pode concluir que exista relação do local com a renda e com nível de escolaridade, pois os entrevistados da Asa Norte onde, em geral, residem pessoas com boa renda e bom nível de escolaridade, relataram que preço está entre importante e muito importante. Por outro lado, os entrevistados do Recanto das Emas onde, em geral residem pessoas de menor poder aquisitivo e com baixa escolaridade, valorizaram o teor de vitamina da mesma forma que os entrevistados da Asa Norte.

A diferença de idade interferiu na pontuação do nível de importância de cor e valor nutricional no preço, teor de vitamina, tamanho, formato e peso, na segunda pesquisa. Na primeira, as pessoas mais jovens demonstraram maior preocupação com a coloração uniforme. Na segunda, os entrevistados com idade entre 51 e 60 anos enfatizaram mais tamanho, formato e peso do pimentão. Em adição, as pessoas acima dos 30 anos consideraram mais o preço quando vão comprar o produto. Em ambas as pesquisas, pessoas com maior faixa etária ressaltaram a necessidade de consumir alimentos com propriedades funcionais. Por último, na esfera do estado civil, as pessoas casadas atentaram mais a preço e, as solteiras, ao peso do produto. Para as demais características, a percepção foi a mesma.

As informações obtidas nessa pesquisa sugerem que os consumidores enfatizam a textura e as características relacionadas ao aspecto quando vão comprar o pimentão. Em adição, foram detectadas diferenças de percepção entre gênero, idade, estado civil, grau de escolaridade e localização do supermercado. Observou-se que os dois últimos itens não são suficientes para explicar os segmentos de renda.

Ressalta-se que essa pesquisa analisou o comportamento de 944 consumidores do Distrito Federal. Mesmo com poucos contrastes nas duas pesquisas sobre o ranqueamento dos atributos, as diferenças de percepções entre as classes estudadas são úteis para estudos de segmentação de consumidores com o intuito de posicionar melhor um novo produto no mercado. 
Tabela 3b. continuação ...(continuation ...)

\begin{tabular}{lccccc}
\hline & Preço & $\begin{array}{c}\text { Valor } \\
\text { nutricional }\end{array}$ & Tamanho & Formato & Peso \\
\hline Sexo & $\mathrm{p}=0,39$ & $\mathrm{p}=0,4519$ & $\mathrm{p}=0,02$ & $\mathrm{p}=0,039$ & $\mathrm{p}=0,075$ \\
\hline Masculino & $3,80 \mathrm{a}$ & $3,81 \mathrm{a}$ & $3,18 \mathrm{a}$ & $3,06 \mathrm{a}$ & $2,78 \mathrm{a}$ \\
Feminino & $3,92 \mathrm{a}$ & $3,70 \mathrm{a}$ & $2,84 \mathrm{~b}$ & $2,77 \mathrm{~b}$ & $2,53 \mathrm{a}$ \\
\hline Grau de escolaridade & $\mathrm{p}=0,01$ & $\mathrm{p}<0,001$ & $\mathrm{p}=0,07$ & $\mathrm{p}=0,03$ & $\mathrm{p}=0,04$ \\
\hline Primeiro grau incompleto & $3,79 \mathrm{bc}$ & $3,17 \mathrm{c}$ & $3,07 \mathrm{ab}$ & $2,78 \mathrm{~b}$ & $2,55 \mathrm{~b}$ \\
Primeiro grau completo & $3,43 \mathrm{c}$ & $3,73 \mathrm{~b}$ & $3,29 \mathrm{a}$ & $3,31 \mathrm{a}$ & $3,06 \mathrm{a}$ \\
Segundo grau completo & $3,96 \mathrm{ab}$ & $3,82 \mathrm{~b}$ & $2,91 \mathrm{ab}$ & $2,87 \mathrm{~b}$ & $2,54 \mathrm{~b}$ \\
Terceiro grau completo & $4,15 \mathrm{a}$ & $4,22 \mathrm{a}$ & $2,69 \mathrm{~b}$ & $2,66 \mathrm{~b}$ & $2,53 \mathrm{~b}$ \\
\hline Idade & $\mathrm{p}=0,009$ & $\mathrm{p}<0,0001$ & $\mathrm{p}=0,08$ & $\mathrm{p}=0,005$ & $\mathrm{p}=0,002$ \\
\hline 31-40 anos & $3,38 \mathrm{~b}$ & $3,67 \mathrm{~b}$ & $2,74 \mathrm{~b}$ & $2,66 \mathrm{~b}$ & $2,38 \mathrm{c}$ \\
41-50 anos & $3,97 \mathrm{a}$ & $3,30 \mathrm{~b}$ & $2,77 \mathrm{~b}$ & $2,63 \mathrm{~b}$ & $2,32 \mathrm{c}$ \\
51-60 anos & $4,14 \mathrm{a}$ & $3,68 \mathrm{~b}$ & $3,14 \mathrm{ab}$ & $3,06 \mathrm{ab}$ & $2,83 \mathrm{ab}$ \\
Mais que 60 anos & $3,98 \mathrm{a}$ & $4,32 \mathrm{a}$ & $3,22 \mathrm{a}$ & $3,24 \mathrm{a}$ & $2,98 \mathrm{a}$ \\
31-40 anos & $3,93 \mathrm{a}$ & $3,51 \mathrm{~b}$ & $2,81 \mathrm{ab}$ & $2,66 \mathrm{~b}$ & $2,45 \mathrm{bc}$ \\
\hline Estado civil & $\mathrm{p}=0,02$ & $\mathrm{p}=0,29$ & $\mathrm{p}=0,16$ & $\mathrm{p}=0,08$ & $\mathrm{p}=0,048$ \\
\hline Solteiro & $3,75 \mathrm{~b}$ & $3,79 \mathrm{a}$ & $2,95 \mathrm{a}$ & $2,98 \mathrm{a}$ & $2,73 \mathrm{a}$ \\
Casado & $4,10 \mathrm{a}$ & $3,61 \mathrm{a}$ & $2,72 \mathrm{a}$ & $2,70 \mathrm{a}$ & $2,42 \mathrm{~b}$ \\
\hline Local & $\mathrm{p}<0,0001$ & $\mathrm{p}<0,0001$ & $\mathrm{p}=0,0003$ & $\mathrm{p}<0,0001$ & $\mathrm{p}<0,0001$ \\
\hline Ceilândia & $3,56 \mathrm{~b}$ & $2,25 \mathrm{c}$ & $2,85 \mathrm{a}$ & $2,39 \mathrm{c}$ & $2,13 \mathrm{c}$ \\
Taguatinga & $3,16 \mathrm{~b}$ & $4,30 \mathrm{a}$ & $3,29 \mathrm{a}$ & $3,45 \mathrm{a}$ & $2,99 \mathrm{a}$ \\
Asa Norte & $4,21 \mathrm{a}$ & $4,43 \mathrm{a}$ & $2,39 \mathrm{~b}$ & $2,47 \mathrm{c}$ & $2,34 \mathrm{bc}$ \\
Riacho Fundo & $4,21 \mathrm{a}$ & $3,39 \mathrm{~b}$ & $3,13 \mathrm{a}$ & $2,93 \mathrm{~b}$ & $2,65 \mathrm{ab}$ \\
Recanto das Emas & $4,25 \mathrm{a}$ & $4,34 \mathrm{a}$ & $3,16 \mathrm{a}$ & $3,16 \mathrm{ab}$ & $3,02 \mathrm{a}$ \\
\hline & & \multicolumn{5}{c}{} &
\end{tabular}

A extrapolação para outros estados necessitará de estudos adicionais para inferir o comportamento dos demais consumidores. Propõe-se, no futuro, realizar um trabalho em outros estados no intuito de comparar e validar a diferença de perfil dos consumidores quanto aos atributos do pimentão.

Por fim, as informações desse estudo apoiarão a pesquisa de melhoramento de Capsicum para desenvolver novas cultivares de pimentão que atendam às exigências dos consumidores preocupados com a firmeza e a aparência, que é um valor cosmético (Reifschneider \& Lopes, em consulta). Considerando o tempo e o investimento necessários nos programas de melhoramento para produzir novas cultivares, é necessário o entendimento da demanda presente dos consumidores para que sejam desenvolvidas cultivares dentro das tendências de consumo, que são temporariamente relevantes.

\section{AGRADECIMENTOS}

Agradecemos aos Drs.Gilmar P.
Henz, Carlos Alberto Lopes e Jadir Borges Pinheiro pela valiosa contribuição neste trabalho; aos gerentes dos supermercados pela colaboração na realização da pesquisa; ao analista Osório Filho pela revisão; e aos estagiários Franque Salviano e Arlysson Barros Ulhoa pelo apoio à realização do trabalho.

\section{REFERÊNCIAS}

ABCSEM. 2007. Pesquisa de mercado de sementes de hortaliças 2007. Disponível em http://www. abcsem.com.br/docs/pesquisa_mercado_2007. pdf. Acessado em 4 de junho de 2009.

ANDREUCCETTI C; FERREIRA MD; GUTIERREZ ASD; TAVARES M. 2005. Caracterização da comercialização de tomate de mesa na CEAGESP: perfil dos atacadistas. Horticultura Brasileira 23: 324-328.

BLACKWELL RD; MINIARD PW; ENGEL JF. 2005. Comportamento do Consumidor. Rio de Janeiro: Cengage Learning. 606 p.

BABBIE E. 2003. Métodos de pesquisas de survey. Belo Horizonte: UFMG. 519p.

CEPA. Centro de Socioeconomia e Planejamento Agrícola. 2009, 4 de junho. Produção Vegetal - Hortaliças, flores e plantas ornamentais. Disponível em http://cepa.epagri.sc.gov.br/ Dados_do_LAC/tabelas_modulo4.3.htm

CICCANTELLI S; MAGIDSON J. 1993.
From experience: Consumer idealized design-involving consumers in the product development process. Journal of Product Innovation Management 10: 341-347.

CLARK K; WHEELRIGHT SC. 1993. Managing New Product and Process Development: Test and Cases. New York: Fee Press. 896 p.

CODEPLAN. 1997. Pesquisa de Informações Sócio-Economicas das Famílias do Distrito Federal - PISEF/DF/97. 120p.

COOPER RG. 1999. From experience: The invisible success factors in product innovation. Journal of Product Innovation Management 16: $115-133$.

DELLA VECCHIA PT. 2007. A indústria de sementes de hortaliças no Brasil. Palestra apresentada no $4^{\circ}$ Congresso de Melhoramento de Plantas. Disponível em http://www.ufla. br/eventos/cbmp/PDF/Paulo\%20Della\%20 Vecchia.pdf. Acessado em 15 de junho de 2009.

FILLION L; KILCAST D. 2002. Consumer perception of crispness and crunchiness in fruits and vegetables. Food Quality and Preference 13: 23-29.

FRANK CA; NELSON RG; SIMONNE EH; BEHE BK; SIMONNE AH. 2001. Consumer preferences for color, price, and vitamin $\mathrm{C}$ content of bell peppers. HortScience 36: 795-800.

GORENSTEIN O; GUTIERREZ ASD. 2000. Esforço de modernização do mercado de frutas e hortaliças no estado de São Paulo. Informações Econômicas 30: 55-60. Disponível em http://www.iea.sp.gov.br/out/ verTexto.php? codTexto $=294$. Acessado em 25 de janeiro de 2010.

HAIR JF; TATHAM RL; ANDERSON RE; BACK W. 2005. Análise Multivariada de Dados. $5^{\text {a }}$ edição. Porto Alegre: Bookman, 593p.

HENZ GP; COSTA CSR; CARVALHO S; BANCI CA. 2007. Negócio rentável. Caderno Técnico da edição da Cultivar HF 42: 1-7.

IEA. Instituto de Economia Agrícola. 2008. Área de produção dos principais produtos da agropecuária do estado de São Paulo. Disponível em http://www.iea.sp.gov.br/out/ banco/menu.php. Acessado em 4 de junho de 2009.

KADER AA. 2002. Postharvest Technology of Horticultural Crops. University of California: Division of Agriculture and Natural Resources Publication 3311.535 p. Disponível em: http:// books.google.com/books?hl=ptBR\&lr=\&id $=$ mDC6ogQT $18 \mathrm{EC} \&$ oi $=$ fnd $\& p g=P A 1 \& d q$ $=$ Postharvest + Technology + of + Horticultura $1+$ Crops\&ots $=$ MzJea3F_5H\&sig $=\mathrm{IR} 0 \mathrm{NrJ} 5 \mathrm{~s}$ bzJe37MBFv8P-2Ks3Es. Acessado em 3 de maio de 2009.

LANA MM; MOITA AW; SOUZA GS; NASCIMENTO EF; MELO MF. Identificação das causas de perdas pós-colheita de pimentão no varejo. Brasília: Embrapa Hortaliças, 2006 (Boletim de Pesquisa e Desenvolvimento Embrapa Hortaliças).23p.

MALDONADO V. 2000. O cultivo do pimentão. Revista Cultivar Hortaliças e Frutas On line. Disponível em http://www.grupocultivar.com. br/artigos/artigo.asp?id=141. Acessado em 15 de junho de 2009. 
MATSUURA FCAU; COSTA JIP; FOLEGATTI MIS. 2004. Marketing de banana: preferências do consumidor quanto aos atributos de qualidade dos frutos. Revista Brasileira de Fruticultura 26: 48-52.

MATTAR FN. 1997. Pesquisa de marketing: metodologia, planejamento. 4.ed. São Paulo: Atlas. 336p.

PEREZ R; RAMOS AM; BINOTI ML; SOUSA PHM; MACHADO GM; CRUZ IB. 2008. Perfil dos consumidores de hortaliças minimamente processadas de Belo Horizonte. Horticultura Brasileira 26: 441-446.

RAGAERT P; VERBEKEB W; DEVLIEGHERE AF; DEBEVERE AJ. 2004. Consumer perception and choice of minimally processed vegetables and packaged fruits. Food Quality and Preference 15: 259-270.

ROJO FJG. 1998. Pesquisa: Comportamento do
Consumidor nos Supermercados. RAE-Revista de Administração de Empresas 38: 16-24.

SAABOR A. 2001. Comercialização de hortaliças sob o enfoque do mercado varejista. Horticultura Brasileira 19: 18.

SCHFFMAN LG; KANUK LL. 2000. Comportamento do consumidor. Rio de Janeiro: PrenticeHall. 492p.

SEAB. Secretaria da Agricultura e do Abastecimento do Paraná. 2009, 4 de junho. Estimativa da área da produção de hortaliças, no Paraná- safras 00/01 a 02/03. Disponível em: www.pr.gov.br/seab/deral/hrtpr.xls

SEAPA. Secretaria de Estado de Agricultura, Pecuária e Abastecimento. 2009, 4 de junho. Pimentão: aumento de qualidade impulsiona vendas. Disponível em http://www.agricultura. mg.gov.br/noticia.asp?id=1247. Acessado em 4 de julho de 2009.
SHETH JN; MITTAL B; NEWMAN BI. 2001. Comportamento do cliente: indo além do comportamento do consumidor. São Paulo: Atlas.

SOUZA RAM. 2005. Mudanças no consumo e na distribuição de alimentos - $O$ caso da distribuição de hortaliças de folhas na cidade de São Paulo. Campinas, UNICAMP-IE. 150p (Tese mestrado).

STOKES ME; DAVIS CS; KOCH GG. 2000. Categorical Data Analysis Using SAS System. Second Edition. Cary, NC: SAS Institute Inc, 623 p.

VAN DER POL M; RYAN M. 1996. Using conjoint analysis to establish consumer preferences for fruit and vegetablesUsing conjoint analysis to establish consumer preferences for fruit and vegetables. British Food Journal 98: 5-12. 